\title{
ON UNIQUENESS AND CONTINUOUS DEPENDENCE IN NONLINEAR THERMODYNAMICS OF ELECTROMAGNETIC MATERIALS
}

\author{
BY \\ D. IEŞAN \\ University of Iaşi, Iaşi, Romania
}

\begin{abstract}
The paper is concerned with the boundary-initial-value problems of the nonlinear thermodynamics of electromagnetic materials. Uniqueness and continuous dependence results are established.
\end{abstract}

1. Introduction. The equations of electromagnetic theory have been the subject of many investigations.

In [1], Pettini has proven a uniqueness theorem for solutions of Maxwell's equations for isotropic materials without thermodynamic influences but with memory. Fabrizio [2] has obtained a broad generalization of this result (see also, Graffi [3, Sec. 2.9]). In the present paper we consider the thermodynamic theory of electromagnetic materials without memory. It concerns rigid materials which conduct both heat and electricity. The purpose of this paper is to establish uniqueness and continuous dependence results in the nonlinear theory. Within the context of classical nonlinear thermoelasticity, Dafermos [4] has established continuous dependence of thermodynamic processes upon initial state and supply terms for materials without heat conduction. These results are based on the local convexity of internal energy and the strong ellipticity condition. In [5], Chiriţă extended the results of Dafermos to heat-conducting materials.

The results established in the present paper are based on the notion of stability used by Dafermos [4], which is equivalent to continuous dependence of thermodynamic processes upon initial state and supply terms. Our analysis is developed both for the materials without heat conduction and for definite conductor of heat materials.

2. Basic equations. We suppose that a properly regular region $R$ of threedimensional Euclidean space $\mathscr{E}_{3}$ is occupied by a rigid body which does not move. Since the configuration of the body is constant in time, there is no reason to distinguish a material point from its place in $\mathscr{E}_{3}$. We let $\bar{R}$ denote the closure of $R$, call $\partial R$ the boundary of $R$, and designate by $\mathbf{n}$ the outward unit normal of $\partial R$. Letters in boldface stand for tensors of an order $p \geq 1$, and if $\mathbf{v}$ has the order $p$, we write

Received August 9, 1988.

(c) 1990 Brown University 
$v_{i j \ldots k}$ ( $p$ subscripts) for the components of $\mathbf{v}$ in the underlying rectangular Cartesian coordinate frame. We shall employ the usual summation and differentiation conventions: the subscripts are understood to range over the integers $(1,2,3)$; summation over repeated subscripts is implied; subscripts preceded by a comma denote partial differentiation with respect to the corresponding Cartesian coordinate; a superposed dot denotes partial differentiation with respect to $t$; the symbol $|\cdot|$ denotes the norm either in an Euclidean vector space or in a tensor space, while $\|\cdot\|$ denotes $L_{2}$-norm.

A thermodynamic process for $R$ is described by a collection of ten functions of place $\mathbf{x}$ and time $t$ (cf. Coleman and Dill $[6,7]$ and McCarthy [8]). These functions are: (1) the electric intensity $\mathbf{E}$, (2) the electric induction $\mathbf{D}$, (3) the magnetic intensity $\mathbf{H}$, (4) the magnetic induction B, (5) the electric current $\mathbf{J},(6)$ the heat flux $\mathbf{q}$, (7) the free-enthalpy $\zeta,(8)$ the entropy density $\eta$, (9) the absolute temperature $\theta>0$, and (10) the heat supply $S$, per unit volume. The first six of these functions are vector-valued and the last four are real-valued. Such a set of ten functions, defined for all $\mathbf{x}$ in $R$, and all $t$ in $\left[0, t_{0}\right]$, is called a thermodynamic process if and only if it is compatible with Maxwell's equations and the equation of balance of energy. Thus, in every thermodynamic process, the following equations must hold (cf. [6,7])

$$
\begin{gathered}
\operatorname{curl} \mathbf{E}=\dot{\mathbf{B}}, \\
\operatorname{curl} \mathbf{H}=\dot{\mathbf{D}}+\mathbf{J}, \\
\dot{\zeta}+\dot{\theta} \eta+\theta \dot{\eta}+\mathbf{D} \cdot \dot{\mathbf{E}}+\mathbf{B} \cdot \dot{\mathbf{H}}=\mathbf{E} \cdot \mathbf{J}+S-\operatorname{div} \mathbf{q} .
\end{gathered}
$$

We do not consider the relations

$$
\operatorname{div} \mathbf{D}=0, \quad \operatorname{div} \mathbf{B}=0,
$$

since we regard these equations as consequences of (2.1), (2.2) and initial conditions.

It is evident that, in order to specify a thermodynamic process it suffices to prescribe the nine functions $\mathbf{E}, \mathbf{D}, \mathbf{H}, \mathbf{B}, \mathbf{J}, \mathbf{q}, \zeta, \eta$, and $\theta$. The function $S$ is then determined by (2.3).

The material at each point $\mathbf{x}$ in $R$ is specified by listing six functions $\hat{\zeta}, \hat{\mathbf{D}}, \hat{\mathbf{B}}, \hat{\eta}$, $\hat{\mathbf{J}}$, and $\hat{\mathbf{q}}$, called constitutive functionals, which give the values taken by $\zeta, \mathbf{D}, \mathbf{B}, \eta$, $\mathbf{J}$, and $\mathbf{q}$ at $(\mathbf{x}, t)$, as functions of the values of $\mathbf{E}, \mathbf{H}, \theta$, and $\mathbf{g}=\operatorname{grad} \theta$ at $(\mathbf{x}, t)$. We consider that $\hat{\zeta}, \hat{\mathbf{D}}, \hat{\mathbf{B}}, \hat{\eta}, \hat{\mathbf{J}}$, and $\hat{\mathbf{q}}$ are smooth functions defined for $\mathbf{E}, \mathbf{H}$, and $\mathbf{g}$ in $\mathscr{E}_{3}, \theta$ in the set of positive real numbers $\mathscr{R}^{+}$, and $\mathbf{x}$ in $\bar{R}$. In particular we assume that the partial derivatives of $\hat{\zeta}, \hat{\mathbf{D}}, \hat{\mathbf{B}}, \hat{\eta}, \hat{\mathbf{J}}$, and $\hat{q}$, at any fixed state $(\mathbf{E}, \mathbf{H}, \theta, \mathbf{g})$ in the space $\mathscr{E}_{3} \times \mathscr{E}_{3} \times \mathscr{R}^{+} \times \mathscr{E}_{3}$, are bounded functions of $(\mathbf{x}, t)$ on $\bar{R} \times\left[0, t_{0}\right]$.

A thermodynamic process is said to be admissible in $R$ if it is compatible with the constitutive equations at each point $\mathbf{x}$ and each time $t$. We shall say that $A=$ $(\mathbf{E}, \mathbf{H}, \theta, \mathbf{g})$ is an admissible state if there exist the functions $\mathbf{D}, \mathbf{B}, \zeta, \eta, \mathbf{q}$, and $\mathbf{J}$ such that $(\mathbf{E}, \mathbf{D}, \mathbf{H}, \mathbf{B}, \mathbf{J}, \mathbf{q}, \zeta, \eta, \theta)$ is an admissible thermodynamic process. The admissible state $A=(\mathbf{E}, \mathbf{H}, \theta, \mathbf{g})$ is called smooth in $R$ if it is such that $\mathbf{E}, \mathbf{H}, \theta$, and $\mathbf{g}$ are Lipschitz continuous, uniformly on bounded subsets of their domain. 
For the Clausius-Duhem inequality to hold for all smooth admissible thermodynamic processes in $R$, it is necessary and sufficient that (cf. [6])

$$
\begin{gathered}
\zeta=\hat{\zeta}(\mathbf{E}, \mathbf{H}, \theta), \\
\mathbf{D}=-\frac{\partial \hat{\zeta}}{\partial \mathbf{E}}, \quad \mathbf{B}=-\frac{\partial \hat{\zeta}}{\partial \mathbf{H}}, \quad \eta=-\frac{\partial \hat{\zeta}}{\partial \theta}, \\
\mathbf{g} \cdot \hat{\mathbf{q}}(\mathbf{E}, \mathbf{H}, \theta, \mathbf{g}) \leq \theta \mathbf{E} \cdot \hat{\mathbf{J}}(\mathbf{E}, \mathbf{H}, \theta, \mathbf{g}) .
\end{gathered}
$$

For smooth admissible thermodynamic processes the balance of energy reduces to

$$
\theta \dot{\eta}=\mathbf{E} \cdot \mathbf{J}-\operatorname{div} \mathbf{q}+S .
$$

3. Preliminary lemmas. Let $A=(\mathbf{E}, \mathbf{H}, \theta, \mathbf{g})(\mathbf{x}, t)$ and $\bar{A}=(\overline{\mathbf{E}}, \overline{\mathbf{H}}, \bar{\theta}, \overline{\mathbf{g}})(\mathbf{x}, t),(\mathbf{x}, t) \in$ $\bar{R} \times\left[0, t_{0}\right]$, be two smooth admissible states. We define

$$
W(t)=\int_{R}[\zeta-\bar{\zeta}+\mathbf{D} \cdot(\mathbf{E}-\overline{\mathbf{E}})+\mathbf{B} \cdot(\mathbf{H}-\overline{\mathbf{H}})+\eta(\theta-\bar{\theta})](\mathbf{x}, t) d \mathbf{x}, \quad t \in\left[0, t_{0}\right],
$$

where

$$
\begin{gathered}
\zeta=\hat{\zeta}(A), \quad \bar{\zeta}=\hat{\zeta}(\bar{A}), \\
\mathbf{D}=-\frac{\partial \hat{\zeta}(A)}{\partial \mathbf{E}}, \quad \mathbf{B}=-\frac{\partial \hat{\zeta}(A)}{\partial \mathbf{H}}, \quad \eta=-\frac{\partial \hat{\zeta}(A)}{\partial \theta} .
\end{gathered}
$$

LemMa 3.1. If $A$ and $\bar{A}$ are two smooth admissible states corresponding to the heat supplies $S$ and $\bar{S}$ in $L^{\infty}\left(R \times\left[0, t_{0}\right]\right)$, then

$$
\begin{aligned}
\dot{W}= & -\int_{\partial R}\left[(\mathbf{E}-\overline{\mathbf{E}}) \times(\mathbf{H}-\overline{\mathbf{H}})+\frac{1}{\theta}(\theta-\bar{\theta})(\mathbf{q}-\overline{\mathbf{q}})\right] \cdot \mathbf{n} d a \\
& +\int_{R} \bar{\theta}(\theta-\bar{\theta})(S-\bar{S}) d \mathbf{x} \\
& +\int_{R} \frac{1}{\theta}(\theta-\bar{\theta})[\overline{\mathbf{J}} \cdot(\mathbf{E}-\overline{\mathbf{E}})+\mathbf{E} \cdot(\mathbf{J}-\overline{\mathbf{J}})-\dot{\bar{\eta}}(\theta-\bar{\theta})] d \mathbf{x} \\
& +\int_{R}\left[\frac{1}{\theta}(\theta-\bar{\theta})\right]_{, i}\left(q_{i}-\bar{q}_{i}\right) d \mathbf{x} \\
& -\int_{R}\left\{\dot{\bar{H}}_{i}\left[B_{i}-\bar{B}_{i}-\frac{\partial B_{i}}{\partial E_{j}}\left(E_{j}-\bar{E}_{j}\right)-\frac{\overline{\partial B_{i}}}{\partial H_{j}}\left(H_{j}-\bar{H}_{j}\right)-\frac{\overline{\partial B_{i}}}{\partial \theta}(\theta-\bar{\theta})\right]\right. \\
& \quad+\dot{\bar{E}}_{i}\left[D_{i}-\bar{D}_{i}-\frac{\overline{\partial D_{i}}}{\partial E_{j}}\left(E_{j}-\bar{E}_{j}\right)-\frac{\overline{\partial D_{i}}}{\partial H_{j}}\left(H_{j}-\bar{H}_{j}\right)-\frac{\overline{\partial D_{i}}}{\partial \theta}(\theta-\bar{\theta})\right] \\
& \quad+\dot{\bar{\theta}}\left[\eta-\bar{\eta}-\frac{\overline{\partial \eta}}{\partial E_{i}}\left(E_{i}-\bar{E}_{i}\right)-\frac{\overline{\partial \eta}}{\partial H_{i}}\left(H_{i}-\bar{H}_{i}\right)-\frac{\overline{\partial \eta}}{\partial \theta}(\theta-\bar{\theta})\right]
\end{aligned}
$$


Proof. In view of (2.3),

$$
\begin{aligned}
F \equiv & \frac{\partial}{\partial t}[\zeta-\bar{\zeta}+\mathbf{D} \cdot(\mathbf{E}-\overline{\mathbf{E}})+\mathbf{B} \cdot(\mathbf{H}-\overline{\mathbf{H}})+\eta(\theta-\bar{\theta})] \\
= & S-\operatorname{div} \mathbf{q}+\mathbf{E} \cdot \mathbf{J}-(\bar{S}-\operatorname{div} \overline{\mathbf{q}}+\overline{\mathbf{E}} \cdot \overline{\mathbf{J}})-\dot{\bar{\theta}}(\eta-\bar{\eta})-\bar{\theta}(\dot{\eta}-\dot{\bar{\eta}}) \\
& +\dot{\overline{\mathbf{D}}} \cdot(\mathbf{E}-\overline{\mathbf{E}})-\dot{\overline{\mathbf{E}}} \cdot(\mathbf{D}-\overline{\mathbf{D}})+\dot{\overline{\mathbf{B}}} \cdot(\mathbf{H}-\overline{\mathbf{H}})-\dot{\mathbf{H}} \cdot(\mathbf{B}-\overline{\mathbf{B}})+(\dot{\mathbf{B}}-\dot{\overline{\mathbf{B}}}) \cdot(\mathbf{H}-\overline{\mathbf{H}}) \\
& +(\dot{\mathbf{D}}-\dot{\overline{\mathbf{D}}}) \cdot(\mathbf{E}-\overline{\mathbf{E}}) .
\end{aligned}
$$

Since

$$
\begin{gathered}
\operatorname{div}(\mathbf{E}-\overline{\mathbf{E}}) \times(\mathbf{H}-\overline{\mathbf{H}})=(\mathbf{H}-\overline{\mathbf{H}}) \cdot \operatorname{curl}(\mathbf{E}-\overline{\mathbf{E}})-(\mathbf{E}-\overline{\mathbf{E}}) \cdot \operatorname{curl}(\mathbf{H}-\overline{\mathbf{H}}) \\
\operatorname{curl}(\mathbf{E}-\overline{\mathbf{E}})=-(\dot{\mathbf{B}}-\dot{\overline{\mathbf{B}}}), \quad \operatorname{curl}(\mathbf{H}-\overline{\mathbf{H}})=\dot{\mathbf{D}}-\dot{\mathbf{D}}+\mathbf{J}-\overline{\mathbf{J}},
\end{gathered}
$$

it follows that

$$
-\operatorname{div}(\mathbf{E}-\overline{\mathbf{E}}) \times(\mathbf{H}-\overline{\mathbf{H}})=(\dot{\mathbf{D}}-\dot{\overline{\mathbf{D}}}) \cdot(\mathbf{E}-\overline{\mathbf{E}})+(\dot{\mathbf{B}}-\dot{\overline{\mathbf{B}}}) \cdot(\mathbf{H}-\overline{\mathbf{H}})+(\mathbf{E}-\overline{\mathbf{E}}) \cdot(\mathbf{J}-\overline{\mathbf{J}}) .
$$

By (2.6),

$$
\begin{aligned}
S-\operatorname{div} \mathbf{q}+\mathbf{E} \cdot \mathbf{J}-\bar{S}+\operatorname{div} \overline{\mathbf{q}}-\overline{\mathbf{E}} \cdot \overline{\mathbf{J}}-\bar{\theta}(\dot{\eta}-\dot{\bar{\eta}})-\dot{\bar{\eta}}(\theta-\bar{\theta}) \\
=\frac{1}{\theta}(\theta-\bar{\theta})[S-\bar{S}+\mathbf{E} \cdot(\mathbf{J}-\overline{\mathbf{J}})+\overline{\mathbf{J}} \cdot(\mathbf{E}-\overline{\mathbf{E}})-\dot{\bar{\eta}}(\theta-\bar{\theta})] \\
-\left[\frac{1}{\theta}(\theta-\bar{\theta})\left(q_{i}-\bar{q}_{i}\right)\right]_{, i}+\left[\frac{1}{\theta}(\theta-\bar{\theta})\right]_{, i}\left(q_{i}-\bar{q}_{i}\right) .
\end{aligned}
$$

It follows from (3.4)-(3.6) that

$$
\begin{aligned}
F= & -\operatorname{div}\left[(\mathbf{E}-\overline{\mathbf{E}}) \times(\mathbf{H}-\overline{\mathbf{H}})+\frac{1}{\theta}(\theta-\bar{\theta})(\mathbf{q}-\overline{\mathbf{q}})\right]+\dot{\overline{\mathbf{D}}} \cdot(\mathbf{E}-\overline{\mathbf{E}}) \\
& -\dot{\overline{\mathbf{E}}} \cdot(\mathbf{D}-\overline{\mathbf{D}})+\dot{\overline{\mathbf{B}}} \cdot(\mathbf{H}-\overline{\mathbf{H}})-\dot{\mathbf{H}} \cdot(\mathbf{B}-\overline{\mathbf{B}})+\dot{\bar{\eta}}(\theta-\bar{\theta})-\dot{\bar{\theta}}(\eta-\bar{\eta}) \\
& -(\mathbf{E}-\overline{\mathbf{E}}) \cdot(\mathbf{J}-\overline{\mathbf{J}})+\frac{1}{\theta}(\theta-\bar{\theta})[S-\bar{S}-\dot{\bar{\eta}}(\theta-\bar{\theta})+\mathbf{E} \cdot(\mathbf{J}-\overline{\mathbf{J}})+\overline{\mathbf{J}} \cdot(\mathbf{E}-\overline{\mathbf{E}})] \\
& +\left[\frac{1}{\theta}(\theta-\bar{\theta})\right]_{, i}\left(q_{i}-\bar{q}_{i}\right) .
\end{aligned}
$$

Next, by (2.4),

$$
\begin{gathered}
\dot{\bar{D}}_{i}=\frac{\partial \bar{D}_{j}}{\partial \bar{E}_{i}} \dot{\bar{E}}_{j}+\frac{\partial \bar{B}_{j}}{\partial \bar{E}_{i}} \dot{H}_{j}+\frac{\partial \bar{\eta}}{\partial \bar{E}_{i}} \dot{\bar{\theta}}, \\
\dot{\bar{B}}_{i}=\frac{\partial \bar{D}_{j}}{\partial \bar{H}_{i}} \dot{\bar{E}}_{j}+\frac{\partial \bar{B}_{j}}{\partial \bar{H}_{i}} \dot{\bar{H}}_{j}+\frac{\partial \bar{\eta}}{\partial \bar{H}_{i}} \dot{\bar{\theta}}, \\
\dot{\bar{\eta}}=\frac{\partial \bar{D}_{j}}{\partial \bar{\theta}} \dot{\bar{E}}_{j}+\frac{\partial \bar{B}_{j}}{\partial \bar{\theta}} \dot{\bar{H}}_{j}+\frac{\partial \bar{\eta}}{\partial \bar{\theta}} \dot{\bar{\theta}}
\end{gathered}
$$

The relations (3.1), (3.4), (3.7), (3.8), and the divergence theorem imply the desired result.

For a heat-conducting material, we have

$$
\begin{aligned}
\hat{q}_{i}(\mathbf{E}, \mathbf{H}, \theta, \mathbf{g})-\hat{q}_{i}(\overline{\mathbf{E}}, \overline{\mathbf{H}}, \bar{\theta}, \overline{\mathbf{g}})= & -\bar{k}_{i j}\left(g_{j}-\bar{g}_{j}\right)-\bar{m}_{i}(\theta-\bar{\theta}) \\
& -\bar{m}_{i j}\left(E_{j}-\bar{E}_{j}\right)-\bar{p}_{i j}\left(H_{j}-\bar{H}_{j}\right) \\
& +o_{i}(|\mathbf{E}-\overline{\mathbf{E}}|+|\mathbf{H}-\overline{\mathbf{H}}|+|\theta-\bar{\theta}|+|\mathbf{g}-\overline{\mathbf{g}}|),
\end{aligned}
$$


where

$$
\begin{aligned}
\bar{k}_{i j}=-\frac{\partial \hat{q}_{i}(\bar{A})}{\partial g_{j}}, \quad \bar{m}_{i} & =-\frac{\partial \hat{q}_{i}(\bar{A})}{\partial \theta}, \quad \bar{m}_{i j}=-\frac{\partial \hat{q}_{i}(\bar{A})}{\partial E_{j}}, \\
\bar{p}_{i j} & =-\frac{\partial \hat{q}_{i}(\bar{A})}{\partial H_{j}} .
\end{aligned}
$$

We say that the smooth admissible state $\bar{A}$ resides in the region of state space where the material is a definite conductor of heat if the tensor $\bar{k}_{i j}$ is positive definite.

LemmA 3.2. Let $\bar{A}=(\overline{\mathbf{E}}, \overline{\mathbf{H}}, \bar{\theta}, \overline{\mathbf{g}})$ be a smooth admissible state on $R \times\left[0, t_{0}\right]$ which resides in the region of state space where the material is a definite conductor of heat. If $A=(\mathbf{E}, \mathbf{H}, \theta, \mathbf{g})$ is any smooth admissible state on $R \times\left[0, t_{0}\right]$ with the property

$$
|\mathbf{E}-\overline{\mathbf{E}}|+|\mathbf{H}-\overline{\mathbf{H}}|+|\theta-\bar{\theta}|+|\mathbf{g}-\overline{\mathbf{g}}|<\delta
$$

where $\delta$ is a positive constant, then there exist the positive constants $\mu_{1}$ and $\mu_{2}$ such that

$$
\begin{aligned}
\int_{R}\left\{\left[\frac{1}{\theta}(\theta-\bar{\theta})\right]_{, i}\left(q_{i}-\bar{q}_{i}\right)\right\}(\mathbf{x}, t) d \mathbf{x} \leq & -\mu_{1}\|(\mathbf{g}-\overline{\mathbf{g}})(\cdot, t)\|_{L^{2}(R)}^{2} \\
& +\mu_{2}\|(\mathbf{E}-\overline{\mathbf{E}}, \mathbf{H}-\overline{\mathbf{H}}, \theta-\bar{\theta})(\cdot, t)\|_{L^{2}(R)}^{2} .
\end{aligned}
$$

Proof. Since $\bar{k}_{i j}$ is positive definite,

$$
\int_{R}\left[\frac{1}{\theta} \bar{k}_{i j}\left(g_{i}-\bar{g}_{i}\right)\left(g_{j}-\bar{g}_{j}\right)\right](\mathbf{x}, t) d \mathbf{x} \geq k_{0}\|(\mathbf{g}-\overline{\mathbf{g}})(\cdot, t)\|_{L^{2}(R)}^{2},
$$

where $k_{0}$ is a positive constant. By (3.9) and (3.13), we have

$$
\begin{aligned}
& \int_{R}\left\{\left[\frac{1}{\theta}(\theta-\bar{\theta})\right]_{, i}\left(q_{i}-\bar{q}_{i}\right)\right\}(\mathbf{x}, t) d \mathbf{x} \\
& \leq-k_{0}\|(\mathbf{g}-\overline{\mathbf{g}})(\cdot, t)\|_{L^{2}(R)}^{2} \\
& \quad+\int_{R}\left\{a(\theta-\bar{\theta})^{2}+b_{i}(\theta-\bar{\theta})\left(g_{i}-\bar{g}_{i}\right)\right. \\
& \quad+C_{j}(\theta-\bar{\theta})\left(E_{j}-\bar{E}_{j}\right)+F_{i j}\left(g_{i}-\bar{g}_{i}\right)\left(E_{j}-\bar{E}_{j}\right) \\
& \left.\quad+G_{i j}\left(g_{i}-\bar{g}_{i}\right)\left(H_{j}-\bar{H}_{j}\right)+L_{j}(\theta-\bar{\theta})\left(H_{j}-\bar{H}_{j}\right)\right\}(\mathbf{x}, t) d \mathbf{x},
\end{aligned}
$$

where

$$
\begin{gathered}
a=\theta^{-2} \bar{m}_{i} \theta_{, i}, \quad b_{i}=\theta^{-2}\left(\bar{k}_{j i} \theta_{, j}-\theta \bar{m}_{i}\right), \quad C_{j}=\theta^{-2} \bar{m}_{i j} \theta_{, i}, \\
F_{i j}=-\bar{m}_{i j} \theta^{-1}, \quad G_{i j}=-\bar{p}_{i j} \theta^{-1}, \quad L_{j}=\theta^{-2} \bar{p}_{i j} \theta_{, i} .
\end{gathered}
$$

By using the Schwarz inequality and the inequality

$$
2 x y \leq x^{2} \alpha^{-2}+y^{2} \alpha^{2}
$$


the relation (3.14) becomes

$$
\begin{aligned}
2 \int_{R}\{ & {\left.\left[\frac{1}{\theta}(\theta-\bar{\theta})\right]_{, i}\left(q_{i}-\bar{q}_{i}\right)\right\}(\mathbf{x}, t) d \mathbf{x} } \\
\leq & \left(-2 k_{0}+\alpha_{1}^{2}+\alpha_{3}^{2}+\alpha_{4}^{2}\right)\|(\mathbf{g}-\overline{\mathbf{g}})(\cdot, t)\|_{L^{2}(R)}^{2} \\
& +\left(\bar{a}+\alpha_{1}^{-2} \bar{b}+\alpha_{2}^{-2} \bar{C}+\alpha_{5}^{-2} \bar{L}\right)\|(\theta-\bar{\theta})(\cdot, t)\|_{L^{2}(R)}^{2} \\
& +\left(\alpha_{2}^{2}+\alpha_{3}^{-2} \bar{F}\right)\|(\mathbf{E}-\overline{\mathbf{E}})(\cdot, t)\|_{L^{2}(\mathbf{R})}^{2} \\
& +\left(\alpha_{5}^{2}+\alpha_{4}^{-2} \bar{G}\right)\|(\mathbf{H}-\overline{\mathbf{H}})(\cdot, t)\|_{L^{2}(\mathbf{R})}^{2},
\end{aligned}
$$

where $\alpha_{1}, \alpha_{2}, \alpha_{3}, \alpha_{4}$, and $\alpha_{5}$ are nonzero arbitrary constants and $\bar{a}=2 \max |a(\mathbf{x}, t)|$, $\bar{b}=\max |\mathbf{b}(\mathbf{x}, t)|, \bar{C}=\max |\mathbf{C}(\mathbf{x}, t)|, \bar{F}=\max |\mathbf{F}(\mathbf{x}, t)|, \bar{G}=\max |\mathbf{G}(\mathbf{x}, t)|, \bar{L}=$ $\max |\mathbf{L}(\mathbf{x}, t)|$.

We choose the arbitrary constants $\alpha_{1}, \alpha_{3}$, and $\alpha_{4}$ so that

$$
2 k_{0}-\alpha_{1}^{2}-\alpha_{3}^{2}-\alpha_{4}^{2}>0 \text {. }
$$

If we define $\mu_{1}$ and $\mu_{2}$ by

$$
\begin{gathered}
2 \mu_{1}=2 k_{0}-\alpha_{1}^{2}-\alpha_{3}^{2}-\alpha_{4}^{2}, \\
2 \mu_{2}=\max \left(\bar{a}+\alpha_{1}^{-2} \bar{b}+\alpha_{2}^{-2} \bar{C}+\alpha_{5}^{-2} \bar{L}, \alpha_{2}^{2}+\alpha_{3}^{-2} \bar{F}, \alpha_{5}^{2}+\alpha_{4}^{-2} \bar{G}\right),
\end{gathered}
$$

then (3.16) implies (3.12).

Within the context of nonlinear thermoelasticity, the above lemma has been established in [5].

4. Nonconductors of heat. A nonconductor of heat is a material for which the functionals $\hat{\mathbf{q}}$ and $\hat{\mathbf{J}}$ are independent of the temperature gradient. For a nonconductor of heat the inequality (2.5) implies that

$$
\hat{\mathbf{q}}=\mathbf{0}, \quad \mathbf{E} \cdot \hat{\mathbf{J}}(\mathbf{E}, \mathbf{H}, \theta) \geq 0 .
$$

We introduce the notations

$$
\begin{gathered}
\bar{\alpha}_{i j}=\frac{\partial \bar{D}_{i}}{\partial \bar{E}_{j}}=-\frac{\partial^{2} \hat{\zeta}(\bar{A})}{\partial E_{i} \partial E_{j}}, \quad \bar{\beta}_{i j}=\frac{\partial \bar{B}_{i}}{\partial \bar{H}_{j}}=-\frac{\partial^{2} \hat{\zeta}(\bar{A})}{\partial H_{i} \partial H_{j}} \\
\bar{\gamma}_{i j}=\frac{\partial \bar{D}_{i}}{\partial \bar{H}_{j}}=\frac{\partial \bar{B}_{j}}{\partial \bar{E}_{i}}=-\frac{\partial^{2} \hat{\zeta}(\bar{A})}{\partial E_{i} \partial H_{j}}, \\
\bar{\xi}_{i}=\frac{\partial \bar{D}_{i}}{\partial \bar{\theta}}=\frac{\partial \bar{\eta}}{\partial \bar{E}_{i}}=-\frac{\partial^{2} \hat{\zeta}(\bar{A})}{\partial E_{i} \partial \theta} \\
\bar{\zeta}_{i}=\frac{\partial \bar{B}_{i}}{\partial \bar{\theta}}=\frac{\partial \bar{\eta}}{\partial \bar{H}_{i}}=\frac{\partial^{2} \hat{\zeta}(\bar{A})}{\partial \bar{H}_{i} \partial \bar{\theta}} \\
\bar{c}=\frac{\partial^{2} \bar{\eta}}{\partial \bar{\theta}^{2}}=-\frac{\partial^{2} \hat{\zeta}(\bar{A})}{\partial \bar{\theta}^{2}}
\end{gathered}
$$

Clearly

$$
\bar{\alpha}_{i j}=\bar{\alpha}_{j i}, \quad \bar{\beta}_{i j}=\bar{\beta}_{j i}, \quad \bar{\gamma}_{i j}=\bar{\gamma}_{j i} .
$$


THEOREM 4.1. Suppose that the material is a nonconductor of heat. Let $\bar{A}=(\overline{\mathbf{E}}, \overline{\mathbf{H}}, \bar{\theta})$ be a smooth admissible state which corresponds to the heat supply $\bar{S} \in L^{\infty}\left(R \times\left[0, t_{0}\right]\right)$. Let $A=(\mathbf{E}, \mathbf{H}, \theta)$ be any smooth admissible state on $R \times\left[0, t_{0}\right]$, which corresponds to the heat supply $S \in L^{\infty}\left(R \times\left[0, t_{0}\right]\right)$, and satisfies the relations

$$
\begin{gathered}
|\mathbf{E}-\overline{\mathbf{E}}|+|\mathbf{H}-\overline{\mathbf{H}}|+|\theta-\bar{\theta}|<\delta_{1} \quad \text { on } R \times\left[0, t_{0}\right], \\
{[(\mathbf{E}-\overline{\mathbf{E}}) \times(\mathbf{H}-\overline{\mathbf{H}})] \cdot \mathbf{n}=0 \quad \text { on } \partial R \times\left[0, t_{0}\right],}
\end{gathered}
$$

where $\delta_{1}$ is a positive constant. Then there exist the positive constants $c_{1}, c_{2}$, and $c_{3}$ such that

$$
\begin{aligned}
\int_{R}\left[\bar{\alpha}_{i j}\right. & \left(E_{i}-\bar{E}_{i}\right)\left(E_{j}-\bar{E}_{j}\right)+\bar{\beta}_{i j}\left(H_{i}-\bar{H}_{i}\right)\left(H_{j}-\bar{H}_{j}\right)+\bar{c}(\theta-\bar{\theta})^{2} \\
& +2 \bar{\gamma}_{i j}\left(E_{i}-\bar{E}_{i}\right)\left(H_{j}-\bar{H}_{j}\right)+2 \bar{\xi}_{i}\left(E_{i}-\bar{E}_{i}\right)(\theta-\bar{\theta}) \\
\quad & \left.\quad+2 \bar{\zeta}_{i}\left(H_{i}-\bar{H}_{i}\right)(\theta-\bar{\theta})\right](\mathbf{x}, t) d \mathbf{x} \\
\leq & c_{1} \|(\mathbf{E}-\overline{\mathbf{E}}, \mathbf{H}-\overline{\mathbf{H}}, \theta-\bar{\theta})\left(\cdot, 0 \|_{L^{2}(R)}^{2}\right. \\
& +c_{2} \int_{0}^{\tau}\|(\mathbf{E}-\overline{\mathbf{E}}, \mathbf{H}-\overline{\mathbf{H}}, \theta-\bar{\theta})(\cdot, t)\|_{L^{2}(R)}^{2} d t \\
& +c_{3} \int_{0}^{\tau}\|(S-\bar{S})(\cdot, t)\|_{L^{2}(R)}\|(\mathbf{E}-\overline{\mathbf{E}}, \mathbf{H}-\overline{\mathbf{H}}, \theta-\bar{\theta})(\cdot, t)\|_{L^{2}(R)} d t,
\end{aligned}
$$

for all $\tau \in[0, s], s \in\left[0, t_{0}\right]$.

Proof. In view of (2.4), (4.1), (4.3) and the Schwarz inequality, it follows from (3.3) that there exist the positive constants $d_{1}$ and $d_{2}$ such that

$$
\begin{aligned}
\dot{W}(t) \leq & d_{1}\|(\mathbf{E}-\overline{\mathbf{E}}, \mathbf{H}-\overline{\mathbf{H}}, \theta-\bar{\theta})(\cdot, t)\|_{L^{2}(R)}^{2} \\
& +d_{2}\|(S-\bar{S})(\cdot, t)\|_{L^{2}(R)}\|(\theta-\bar{\theta})(\cdot, t)\|_{L^{2}(R)} .
\end{aligned}
$$

We fix $s \in\left[0, t_{0}\right]$ and integrate (4.7) over $[0, \tau], \tau \in[0, s]$. We get

$$
\begin{aligned}
W(\tau) \leq & W(0)+d_{1} \int_{0}^{\tau}\|(\mathbf{E}-\overline{\mathbf{E}}, \mathbf{H}-\overline{\mathbf{H}}, \theta-\bar{\theta})(\cdot, t)\|_{L^{2}(R)}^{2} d t \\
& +d_{2} \int_{0}^{\tau}\|(S-\bar{S})(\cdot, t)\|_{L^{2}(R)}\|(\mathbf{E}-\overline{\mathbf{E}}, \mathbf{H}-\overline{\mathbf{H}}, \theta-\bar{\theta})(\cdot, t)\|_{L^{2}(R)}^{2} d t .
\end{aligned}
$$

By (2.4),

$$
\zeta-\bar{\zeta}+\mathbf{D} \cdot(\mathbf{E}-\overline{\mathbf{E}})+\mathbf{B} \cdot(\mathbf{H}-\overline{\mathbf{H}})+\eta(\theta-\bar{\theta})=\frac{1}{2} \Lambda+o\left(|\mathbf{E}-\overline{\mathbf{E}}|^{2}+|\mathbf{H}-\overline{\mathbf{H}}|^{2}+|\theta-\bar{\theta}|^{2}\right),
$$

where

$$
\begin{aligned}
\Lambda= & \bar{\alpha}_{i j}\left(E_{i}-\bar{E}_{i}\right)\left(E_{j}-\bar{E}_{j}\right)+\bar{\beta}_{i j}\left(H_{i}-\bar{H}_{i}\right)\left(H_{j}-\bar{H}_{j}\right)+\bar{c}(\theta-\bar{\theta})^{2} \\
& +2 \gamma_{i j}\left(E_{i}-\bar{E}_{i}\right)\left(H_{j}-\bar{H}_{j}\right)+2 \bar{\xi}_{i}\left(E_{i}-\bar{E}_{i}\right)(\theta-\bar{\theta}) \\
& +2 \bar{\zeta}_{i}\left(H_{i}-\bar{H}_{i}\right)(\theta-\bar{\theta}) .
\end{aligned}
$$

It follows from (3.1), (4.8)-(4.10) that

$$
\begin{aligned}
\int_{R} \Lambda(\mathbf{x}, \tau) d \mathbf{x} \leq & 2 W(0)+2 d_{1} \int_{0}^{\tau}\|(\mathbf{E}-\overline{\mathbf{E}}, \mathbf{H}-\overline{\mathbf{H}}, \theta-\bar{\theta})(\cdot, t)\|_{L^{2}(R)}^{2} d t \\
& +2 d_{2} \int_{0}^{\tau}\|(S-\bar{S})(\cdot, t)\|_{L^{2}(R)}\|(\mathbf{E}-\overline{\mathbf{E}}, \mathbf{H}-\overline{\mathbf{H}}, \theta-\bar{\theta})(\cdot, t)\|_{L^{2}(R)} d t .
\end{aligned}
$$


Clearly, there exists the positive constant $d_{3}$ such that

$$
W(0) \leq d_{3}\|(\mathbf{E}-\overline{\mathbf{E}}, \mathbf{H}-\overline{\mathbf{H}}, \theta-\bar{\theta})(\cdot, 0)\|_{L^{2}(R)}^{2} .
$$

From (4.11) and (4.12) we obtain the desired result.

We say that the smooth admissible state $\bar{A}=(\overline{\mathbf{E}}, \overline{\mathbf{H}}, \bar{\theta})(\mathbf{x}, t)$ is positive if, for each $(\mathbf{x}, t) \in R \times\left[0, t_{0}\right]$, there exists a positive constant $d$ such that

$$
\bar{\alpha}_{i j} u_{i} u_{j}+\bar{\beta}_{i j} v_{i} v_{j}+\bar{c} w^{2}+2 \bar{\gamma}_{i j} u_{i} v_{j}+2 \bar{\xi}_{i} u_{i} w+2 \bar{\zeta}_{i} v_{i} w \geq d\left(|\mathbf{u}|^{2}+|\mathbf{v}|^{2}+w^{2}\right)
$$

for all vectors $\mathbf{u}, \mathbf{v} \in \mathscr{C}_{3}$ and every $w \in \mathscr{R}$.

If (4.13) holds then the specific heat $\bar{c}$ is strictly positive. Moreover, if $\hat{B}$ is independent of $\mathbf{E}$, then (4.13) implies that the tensor $\bar{\beta}_{i j}$ is positive definite (see, for example, Graffi [3], Sec. 2.4).

The convexity of thermodynamical potentials for electromagnetic materials has been studied by Fabrizio (see $[9,10]$ ).

In what follows we shall need the Gronwall-type inequality [4].

LEMMA 4.1. Assume that the nonnegative functions $y \in L^{\infty}[0, s]$ and $g \in L^{1}[0, s]$ satisfy the inequality

$$
y^{2}(\tau) \leq M^{2} y^{2}(0)+\int_{0}^{\tau}\left[(2 \alpha+4 \beta \tau) y^{2}(t)+2 N g(t) y(t)\right] d t, \quad \tau \in[0, s],
$$

with $\alpha, \beta, M$, and $N$ nonnegative constants. Then

$$
y(s) \leq M \exp \left(\rho s+\beta s^{2}\right) y(0)+N \exp \left(\rho s+\beta s^{2}\right) \int_{0}^{s} g(t) d t,
$$

where $\rho=\alpha+\beta / \alpha$.

We have the following continuous dependence result.

THEOREM 4.2. Suppose that the material is a nonconductor of heat. Let $\bar{A}=(\overline{\mathbf{E}}, \overline{\mathbf{H}}, \bar{\theta})$ and $A=(\mathbf{E}, \mathbf{H}, \theta)$ be as in Theorem 4.1. If $\bar{A}$ is positive then there exist the positive constants $\delta_{1}, \alpha_{1}, M_{1}$, and $N_{1}$ with the property that whenever (4.4) holds, we have

$$
\begin{aligned}
\|(\mathbf{E}-\overline{\mathbf{E}}, \mathbf{H}-\overline{\mathbf{H}}, \theta-\bar{\theta})(\cdot, s)\|_{L^{2}(R)} \\
\leq[\exp ]\left(\alpha_{1} s\right)\left\{M_{1}\|(\mathbf{E}-\overline{\mathbf{E}}, \mathbf{H}-\overline{\mathbf{H}}, \theta-\bar{\theta})(\cdot, 0)\|_{L^{2}(R)}\right. \\
\left.+N_{1} \int_{0}^{2}\|(S-\bar{S})(\cdot, t)\|_{L^{2}(R)} d t\right\},
\end{aligned}
$$

for any $s \in\left[0, t_{0}\right]$.

Proof. In view of (4.6) and (4.13), there exist the positive constants $M_{1}, \alpha_{1}$, and $N_{1}$ such that

$$
\begin{aligned}
& \|(\mathbf{E}-\overline{\mathbf{E}}, \mathbf{H}-\overline{\mathbf{H}}, \theta-\bar{\theta})(\cdot, t)\|_{L^{2}(R)}^{2} \\
& \leq \quad M_{1}^{2}\|(\mathbf{E}-\overline{\mathbf{E}}, \mathbf{H}-\overline{\mathbf{H}}, \theta-\bar{\theta})(\cdot, 0)\|_{L^{2}(R)}^{2} \\
& \quad+\int_{0}^{\tau}\left\{2 \alpha_{1}\|(\mathbf{E}-\overline{\mathbf{E}}, \mathbf{H}-\overline{\mathbf{H}}, \theta-\bar{\theta})(\cdot, t)\|_{L^{2}(R)}^{2}\right. \\
& \left.\quad+2 N_{1}\|(S-\bar{S})(\cdot, t)\|_{L^{2}(R)}\|(\mathbf{E}-\overline{\mathbf{E}}, \mathbf{H}-\overline{\mathbf{H}}, \theta-\bar{\theta})\|_{L^{2}(R)}\right\} d t .
\end{aligned}
$$

The application of Lemma 4.1 leads to (4.16).

The next uniqueness theorem is a direct consequence of Theorem 4.2. 
TheOREM 4.3. Let $A=(\mathbf{E}, \mathbf{H}, \theta)$ and $\bar{A}=(\bar{E}, \bar{H}, \bar{\theta})$ be as in Theorem 4.1. If the corresponding heat supplies $S$ and $\bar{S}$ coincide on $\bar{R} \times\left[0, t_{0}\right]$ and the both states originate from the same initial data, then $\mathbf{E}=\overline{\mathbf{E}}, \mathbf{H}=\overline{\mathbf{H}}, \theta=\bar{\theta}$.

5. Conductors of heat. For a heat-conducting material we have the following result, similar to that given in Sec. 4 .

THEOREM 5.1. Let $\bar{A}=(\overline{\mathbf{E}}, \overline{\mathbf{H}}, \bar{\theta}, \overline{\mathbf{g}})$ be a smooth admissible state on $R \times\left[0, t_{0}\right]$ which resides in the region of state space where the material is a definite conductor of heat and corresponds to the heat supply $\bar{S} \in L^{\infty}\left(R \times\left[0, t_{0}\right]\right)$. Let $A=(\mathbf{E}, \mathbf{H}, \theta, \mathbf{g})$ be any smooth admissible state on $R \times\left[0, t_{0}\right]$ which corresponds to the heat supply $S \in L^{\infty}\left(R \times\left[0, t_{0}\right]\right)$ and satisfies the relations

$$
\begin{gathered}
|\mathbf{E}-\overline{\mathbf{E}}|+|\mathbf{H}-\overline{\mathbf{H}}|+|\theta-\bar{\theta}|+|\mathbf{g}-\overline{\mathbf{g}}|<\delta_{2} \quad \text { on } R \times\left[0, t_{0}\right], \\
{[(\mathbf{E}-\overline{\mathbf{E}}) \times(\mathbf{H}-\overline{\mathbf{H}})] \cdot \mathbf{n}=0, \quad(\theta-\bar{\theta})(\mathbf{q}-\overline{\mathbf{q}}) \cdot \mathbf{n}=0 \quad \text { on } \partial R \times\left[0, t_{0}\right],}
\end{gathered}
$$

where $\delta_{2}$ is a positive constant. If $\bar{A}$ is positive then there exist the positive constants $\alpha_{2}, M_{2}$, and $N_{2}$ with the property that whenever (5.2) holds, we have

$$
\begin{array}{r}
\|(\mathbf{E}-\overline{\mathbf{E}}, \mathbf{H}-\overline{\mathbf{H}}, \theta-\bar{\theta})(\cdot, s)\|_{L^{2}(R)} \\
\leq\left\{\exp \left(\alpha_{2} s\right)\right\}\left[M_{2}\|(\mathbf{E}-\overline{\mathbf{E}}, \mathbf{H}-\overline{\mathbf{H}}, \bar{\theta}-\bar{\theta})(\cdot, 0)\|_{L^{2}(R)}\right. \\
\left.\quad+N_{2} \int_{0}^{s}\|(S-\bar{S})(\cdot, t)\|_{L^{2}(R)} d t\right],
\end{array}
$$

for any $s \in\left[0, t_{0}\right]$.

Proof. In view of Lemmas 3.1, 3.2, there exist the positive constants $m_{1}$ and $m_{2}$ such that, whenever (3.11) holds, we have

This relation implies

$$
\begin{aligned}
\dot{W}(t) \leq & m_{1}\|(\mathbf{E}-\overline{\mathbf{E}}, \mathbf{H}-\overline{\mathbf{H}}, \theta-\bar{\theta})(\cdot, t)\|_{L^{2}(R)}^{2} \\
& +m_{2}\|(S-\bar{S})(\cdot, t)\|_{L^{2}(R)}\|(\theta-\bar{\theta})(\cdot, t)\|_{L^{2}(R)}
\end{aligned}
$$

$$
\begin{aligned}
W(\tau) \leq W(0)+\int_{0}^{\tau}\{ & m_{1}\|(\mathbf{E}-\overline{\mathbf{E}}, \mathbf{H}-\overline{\mathbf{H}}, \theta-\bar{\theta})(\cdot, t)\|_{L^{2}(R)}^{2} \\
& \left.+m_{2}\|(S-\bar{S})(\cdot, t)\|_{L^{2}(R)}\|(\theta-\bar{\theta})(\cdot, t)\|_{L^{2}(R)}\right\} d t, \quad \tau \in[0, s] .
\end{aligned}
$$

As in Sec. 4, we conclude that there exists a positive constant $\delta_{1}$ such that whenever (4.4) holds, the inequality (4.11) is satisfied. In (5.1) we take $\delta_{2}=\min \left(\delta_{1}, \delta\right)$, so that (4.11) and (5.4) hold. In view of (4.12) and (4.13) we conclude that there exist the positive constants $M_{2}, \alpha_{2}$, and $N_{2}$ such that

$$
\begin{aligned}
& \|(\mathbf{E}-\overline{\mathbf{E}}, \mathbf{H}-\overline{\mathbf{H}}, \theta-\bar{\theta})(\cdot, t)\|_{L^{2}(R)}^{2} \\
& \leq M^{2}\|(\mathbf{E}-\overline{\mathbf{E}}, \mathbf{H}-\overline{\mathbf{H}}, \theta-\bar{\theta})(\cdot, 0)\|_{L^{2}(R)}^{2} \\
& \quad+\int_{0}^{\tau}\left\{2 \alpha_{2}\|(\mathbf{E}-\overline{\mathbf{E}}, \mathbf{H}-\overline{\mathbf{H}}, \theta-\bar{\theta})(\cdot, t)\|_{L^{2}(R)}^{2}\right. \\
& \left.\quad+2 N_{2}\|(S-\bar{S})(\cdot, t)\|_{L^{2}(R)}\|(\mathbf{E}-\overline{\mathbf{E}}, \mathbf{H}-\overline{\mathbf{H}}, \theta-\bar{\theta})(\cdot, t)\|_{L^{2}(R)}^{2}\right\} d t .
\end{aligned}
$$

The application of Lemma 4.1 implies the desired result.

An immediate consequence of Theorem 5.1 is the following uniqueness result. 
TheOREM 5.2. Let $A=(\mathbf{E}, \mathbf{H}, \theta, \mathbf{g})$ and $\bar{A}=(\overline{\mathbf{E}}, \overline{\mathbf{H}}, \bar{\theta}, \overline{\mathbf{g}})$ be as in Theorem 5.1. If $A$ and $\bar{A}$ correspond to the same heat supply and they originate from the same initial data, then $\mathbf{E}=\overline{\mathbf{E}}, \mathbf{H}=\overline{\mathbf{H}}, \theta=\bar{\theta}$.

Acknowledgment. The results described here were obtained while the author was a visiting professor at the Catania University. Support from the Consiglio Nazionale delle Ricerche (Italy) is gratefully acknowledged.

\section{REFERENCES}

[1] G. Pettini, Sul teorema di unicità nell'elettromagnetismo nonlineare ereditario, Boll. Un. Mat. Ital., 55-64 (1974)

[2] M. Fabrizio, Problemi di unicità per le equazioni funzionali non lineari del campo elettromagnetico I, II, Rend. Acc. Naz. Lincei 49, I:200-207, II:268-271 (1970)

[3] D. Graff, Nonlinear Partial Differential Equations in Physical Problems, Pitman, Boston-LondonMelbourne, 1980

[4] C. M. Dafermos, The second law of thermodynamics and stability, Arch. Rational Mech. Anal. 70, 167-179 (1979)

[5] S. Chiriță, Uniqueness and continuous data dependence in dynamical problems of nonlinear thermoelasticity, J. Thermal Stresses 5, 331-346 (1982)

[6] B. D. Coleman and E. H. Dill, Thermodynamical restrictions on the constitutive equations of electromagnetic theory, Z. Angew. Math. Phys. 22, 691-702 (1971)

[7] B. D. Coleman and E. H. Dill, On the thermodynamics of electromagnetic fields in materials with memory, Arch. Rational Mech. Anal. 41, 132-162 (1971)

[8] M. F. McCarthy, Thermodynamics of electromagnetic materials with memory, Arch. Rational Mech. Anal. 41, 333-353 (1971)

[9] M. Fabrizio, Sulla convessità dei potenziali termodinamici per materiali con memoria, Ann. Mat. Pura Appl. 101, 33-48 (1974)

[10] M. Fabrizio, Théorèmes d'unicité sur un nouveau problème au limites relatif aux équations non linéaires de Maxwell, J. Mécanique 15, 681-695 (1976) 